\title{
Clinical significance of intra-tumoral sinusoidal structures showing lympho-endothelial immunoreactivity in breast cancer
}

\author{
KENJI TEZUKA ${ }^{1}$, NAOYOSHI ONODA ${ }^{1}$, TSUTOMU TAKASHIMA ${ }^{1}$, TETSURO ISHIKAWA ${ }^{1}$, \\ TOMOKO WAKASA ${ }^{2}$, KENICHI WAKASA $^{2}$ and KOSEI HIRAKAWA ${ }^{1}$ \\ ${ }^{1}$ Department of Surgical Oncology, Osaka City University Graduate \\ School of Medicine; ${ }^{2}$ Department of Diagnostic Pathology, Osaka City \\ University Hospital, 1-4-3 Asahi-machi, Abeno-ku, Osaka 545-8585, Japan
}

Received January 29, 2008; Accepted March 20, 2008

\begin{abstract}
The lymphatic system is known as one of the most important pathways in the disease progression of breast cancer. In this study, we investigated lymphatic systems intratumorally, and found a unique structure displaying lymphoendothelial immunoreactivity. These incomplete vessel-like structures that formed sinusoids and specifically reacted with the D2-40 antibody, were found in the tumor stroma and were often infiltrated by cancer cells. To show the clinical significance of these intra-tumoral sinusoidal structures (ISS), we examined 113 specimens of surgically resected breast cancer. In multivariate logistic regression analysis, lymph node status $(\mathrm{p}=0.024)$ and disease recurrence $(\mathrm{p}=0.008)$ were the independent factors that correlated with the existence of ISS. Positive ISS detection in the tumor correlated more strongly with lymph node metastases than positive lymphovascular invasion by cancer cells. Furthermore, a significant correlation with frequent recurrence and poorer survival were recognized in patients with ISS $(\mathrm{p}<0.001, \log$-rank test). The evaluation of ISS by needle-biopsied specimens was as accurate as that with surgical specimens, and a significant correlation between the positive ISS evaluated by biopsy and positive lymph node status $(\mathrm{p}=0.020)$ was found. In conclusion, ISS are unique structures which play a crucial role in disease progression through lymphatic systems in breast cancer. In a clinical setting, the detection of ISS by biopsy specimen should be considered as a method for determining prognosis instead of a whole tumor examination.
\end{abstract}

Correspondence to: Dr Naoyoshi Onoda, Department of Surgical Oncology, Osaka City University Graduate School of Medicine, 1-4-3 Asahi-machi, Abeno-ku, Osaka 545-8585, Japan

E-mail: nonoda@med.osaka-cu.ac.jp

Key words: breast cancer, D2-40, intra-tumoral lymphatic, lymphogenous metastasis, prognosis

\section{Introduction}

Lymphatic as well as blood vessels are considered to be one of the most important pathways in the disease progression of breast cancer. Hematological metastasis to distant organs is found in $7 \%$ of cases of breast cancer at the initial diagnosis (1). In contrast, metastasis to regional lymph nodes, most commonly to the ipsilateral axillary nodes, is found much more often, in $\sim 45 \%$ of the cases (2). Cases with exclusive lymph node metastases are at high risk for disease recurrence and display poor prognosis (3), as with cases having distant metastases $(4,5)$. Intensive systemic chemotherapy or hormonal therapies are, therefore, more important than surgical resection of the regional lymph node (6). The necessity of additional systemic therapy, before or after surgery, for cases with apparent lymph node metastasis, or with histological evidence of lympho-vascular invasion (LVI) has reached a worldwide consensus (7).

The ability of tumor cells to induce the growth of intratumoral novel lymphatic vessels (lymphangiogenesis), and the link between this lymphangiogenesis and metastasis via the lymphatic system has been demonstrated in basic studies (8). Vascular endothelial growth factor (VEGF)-C plays a central role in this pathway. Some studies, using clinical breast cancer samples, have also shown a correlation between VEGF-C mRNA expression and lymphatic microvessel density (LVD) in the tumor, disease progression (9) and poor prognosis $(10,11)$. However, at the same time, the LVD values found in these studies are scattered compared with blood microvascular density (MVD) values, and with LVD values in normal breast tissue (12). In general, lymphatic systems are hardly observed in tumors, and no evidence of intra-tumoral lymphangiogenesis has been reported in the clinical samples of breast cancer (13-17). Lymphatic vessels found occasionally in tumors have been considered to be embedded with tumor stroma and pre-existing duct or lobes (18) and have been shown to be non-proliferative (13). Furthermore, LVI has rarely been found in tumors, even in studies reporting the significance of the induction of lymphangiogenesis $(9,11)$. Therefore, the existence of a functional structure and the 
clinical significance of an intra-tumoral lymphatic system in breast cancer need further elucidation.

Studies of the lymphatic systems have greatly depended on the recent developments of specific markers for lymphatic vessels. In our previous report, we demonstrated the clinical significance of detecting LVI using D2-40, one of the markers that specifically reacts with lymphatic vessels (19-21), compared to the conventional method by H\&E staining $(16,17)$. We were able to delineate lymphatic vessels more clearly and accurately by immunostaining than by H\&E staining. Moreover, we found a unique intra-tumoral sinusoidal structure that specifically reacts with D2-40 that could not have been found with a conventional H\&E stain. In this study, we investigated this D2-40-reactive intra-tumoral sinusoidal structure (ISS) in terms of its histological and clinical aspects, and found a significant link between ISS and the lymphatic spread of breast cancer.

\section{Materials and methods}

Patients. The study included 131 women with breast cancer, diagnosed and treated in the Osaka City University Hospital, Osaka, Japan, between 1997 and 2000. Additional core needle biopsy (CNB) was performed in 12 patients between November 2005 and March 2006. These patients were added to estimate the correlation of lymph node status or postoperative specimens. All patients had received a mastectomy or conservative breast operation with axillary lymph node dissection. The women who had undergone a conservative breast operation received postoperative radiotherapy to the residual ipsilateral breast. Each patient was treated with suitable adjuvant therapy (chemotherapy or endocrine therapy or none) postoperatively, according to the stage of the disease.

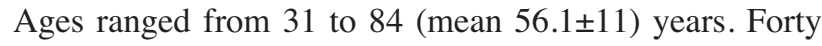
patients $(30.5 \%)$ were $<50$ years, the tumors were $6-90$ (average $28.6 \pm 15.6) \mathrm{mm}$ in diameter, and 67 patients $(51.1 \%)$ had lymph node metastasis at the time of surgery. One hundred and twenty-two cases were invasive ductal carcinoma, 6 cases were invasive lobular carcinoma, 1 case was medullary carcinoma and 2 cases were non-invasive ductal carcinoma. Mucinous and apocrine carcinomas were excluded from this study. Fifty-two patients $(39.7 \%)$ had relapsed disease from the time of the last follow-up. Twenty-eight patients $(21.4 \%)$ with breast cancer had a short survival. The overall 5-year survival and disease-free survival rates after the initial surgical treatment was 81.7 and $59.5 \%$, respectively, with a mean follow-up of $69 \pm 25$ months. Patient characteristics and pathological information were obtained from their clinical records. The pathological features of the tumors had been determined from a standard H\&E-based observation by two pathologists and had been recorded. Written informed consent was obtained from each patient, and the protocol was approved by the ethics committee of Osaka City University.

Immunohistochemistry. Tissues were fixed in $10 \%$ neutral buffered formalin immediately after surgical resection and embedded in paraffin using standard protocols. IHC was performed as previously reported $(4,5)$ on a single representative block from each case, including cancer and noncancerous adjacent tissue. Tissue sections $(4 \mu \mathrm{m})$ were dewaxed and antigen retrieval was performed by autoclaving at $121^{\circ} \mathrm{C}$ for $5 \mathrm{~min}$ in $10 \mathrm{mM}$ citrate buffer (pH 6). Sections were incubated for $25 \mathrm{~min}$ in $3 \%$ hydrogen peroxide to quench endogenous tissue peroxidase. Primary monoclonal antibodies directed against lympho-endothelium were D2-40 (prediluted, Nichirei, Tokyo, Japan) and vascular endothelial growth factor (VEGF)-C (1:150 dilution, Zymed Laboratories, South San Francisco, CA, USA), respectively. Other antibodies used were CD34 (prediluted, Nichirei), S100 (1:400 dilution, Dako Cytomation, Carpinteria, CA), SMA (1:100 dilution, Dako Cytomation) and Flt-4 (1:150 dilution, R\&D Systems, Inc., Minneapolis, MN) to detect blood vessels, nerves, musculature and VEGF-C receptors, respectively. Tissue sections were incubated with $\mathrm{D} 2-40$ for $70 \mathrm{~min}$ at room temperature and with VEGF-C overnight at $4^{\circ} \mathrm{C}$ in a humidified chamber. After washing in phosphate-buffered saline, tissues were incubated with horseradish peroxidase-conjugated anti-rabbit or anti-mouse Ig polymer as a second antibody (Envision kit, Dako Cytomation) for $30 \mathrm{~min}$ at room temperature, according to the manufacturer's instructions. After visualizing the immunoreaction with 3,3'-diaminobenzidine, the sections were counter-stained with hematoxylin. The samples were examined by two investigators without any knowledge of the patient clinical characteristics. LVI was defined as previously described $(16,17)$. The samples with positive immunoreactivity against VEGF-C in $>10 \%$ of cancer cells were considered positive for VEGF-C expression.

Statistical analysis. Statistical analysis was performed with the SPSS 12.0 software package (SPSS, Tokyo, Japan). Differences were considered statistically significant for $\mathrm{p}<0.05$. Correlations between the results with clinicopathological factors were analyzed by $\chi^{2}$ or Fisher's exact test when the assumptions of the $\chi^{2}$ test were not met. Survival curves for patients were calculated using the Kaplan-Meier method and analyzed by the log-rank test. Multivariate logistic regression analysis was performed to identify predictors of recurrence. For each variable, the odds ratio (OR) and $95 \%$ confidence interval $(95 \% \mathrm{CI})$ were calculated.

\section{Results}

The expression of the intra-tumoral sinusoidal structure, immunoreactive to D2-40. As described in our previous report, we have observed D2-40 positive lymphatic vessels in the peritumoral area $(16,17)$. It is extremely rare to observe D2-40 positive lymphatic vessels in the intra-tumoral fibrotic area. In some cases, however, immune reactive cells arranged with an imperfect lumen and with indistinct borders with surrounding structures, including cancer nests and stroma, were displayed (Fig. 1a). They consisted of small, deformed, various-sized sinusoids, lined with D2-40 positive endothelia or incomplete lymphatic endothelial cells, insufficiently developed to form vessels (Fig. 1b). Infiltrations into cancer cells were commonly observed in this structure. We defined this structure as an intra-tumoral sinusoidal structure showing lympho-endothelial immunoreactivity (ISS) in the present study. In contrast to LVI, ISS was hardly observed outside the tumor nests. The majority of the areas of ISS were multi-focal and $>1 \mathrm{~mm}$ in diameter. Although it was impossible to distinguish ISS from 
Table I. Correlation between ISS and clinicopathological variables.

\begin{tabular}{|c|c|c|c|c|c|c|}
\hline \multirow[b]{3}{*}{ Variable } & \multicolumn{3}{|c|}{ Univariate analysis } & \multicolumn{3}{|c|}{ Multivariate analysis } \\
\hline & \multicolumn{2}{|c|}{ ISS } & \multirow[b]{2}{*}{ p-value } & \multirow[b]{2}{*}{ Odds ratio } & \multirow[b]{2}{*}{$95 \% \mathrm{CI}$} & \multirow[b]{2}{*}{ p-value } \\
\hline & No & Yes & & & & \\
\hline \multicolumn{7}{|l|}{ Age } \\
\hline$\leq 50$ & 20 & 20 & \multirow[b]{2}{*}{ NS } & 0.574 & \multirow[t]{2}{*}{$0.115-2.869$} & \multirow[t]{2}{*}{ NS } \\
\hline$>50$ & 50 & 41 & & & & \\
\hline \multicolumn{7}{|l|}{ Tumor size } \\
\hline$\leq 20 \mathrm{~mm}$ & 33 & 12 & \multirow[b]{2}{*}{0.001} & 1.378 & \multirow[t]{2}{*}{$0.512-3.907$} & \multirow[t]{2}{*}{ NS } \\
\hline$>20 \mathrm{~mm}$ & 37 & 49 & & & & \\
\hline \multicolumn{7}{|l|}{ Menopause } \\
\hline No & 18 & 20 & \multirow[b]{2}{*}{ NS } & 0.419 & \multirow[t]{2}{*}{$0.444-12.870$} & \multirow[t]{2}{*}{ NS } \\
\hline Yes & 52 & 41 & & & & \\
\hline \multicolumn{7}{|c|}{ Lymph node metastasis } \\
\hline Negative & 47 & 17 & \multirow{2}{*}{$<0.001$} & 2.841 & \multirow{2}{*}{$0.142-0.871$} & \multirow[t]{2}{*}{0.024} \\
\hline Positive & 23 & 44 & & & & \\
\hline \multicolumn{7}{|l|}{ ER } \\
\hline Negative & 29 & 35 & \multirow[b]{2}{*}{0.05} & 0.511 & \multirow[t]{2}{*}{$0.829-4.625$} & \multirow[t]{2}{*}{ NS } \\
\hline Positive & 41 & 26 & & & & \\
\hline \multicolumn{7}{|c|}{ Nuclear grade } \\
\hline 1 & 62 & 49 & & 0.816 & $0.394-3.805$ & NS \\
\hline 2 or 3 & 8 & 12 & NS & & & \\
\hline HE-LVI & & & & & & \\
\hline- & 39 & 27 & & 1.489 & $0.577-3.841$ & NS \\
\hline+ & 31 & 34 & NS & & & \\
\hline D2-LVI & & & & & & \\
\hline- & 42 & 36 & & 2.427 & $0.145-1.176$ & NS \\
\hline+ & 28 & 25 & NS & & & \\
\hline VEGF-C & & & & & & \\
\hline- & 33 & 10 & & 2.571 & $0.150-1.010$ & NS \\
\hline+ & 37 & 51 & $<0.001$ & & & \\
\hline Recurrence & & & & & & \\
\hline No & 56 & 25 & & 3.831 & 0.096-0.708 & 0.008 \\
\hline Yes & 14 & 36 & $<0.001$ & & & \\
\hline
\end{tabular}

non-immunoreactive sinusoidal tumor stroma morphologically with conventional H\&E-stained specimens, ISS was easily observed by IHC. For the assessment of ISS on immunohistochemistry, it was just deemed present or absent. As a result, ISS was found in 61 out of 131 cases of breast cancer. ISS was observed in biopsy specimens taken with a core needle (Fig. 1c). Fig. 2 shows a schematic figure of the ISS. We performed additional immunostaining with CD34, S-100, SMA and Flt-4 to prove the specificity of this D2-40 positive structure (Fig. 3). Symmetric 'mirror image' specimens were made from the same surface and stained. The area of ISS was unreactive with CD34, S100 or Flt-4. Although tumor stroma were diffusely stained with SMA, the immunoreactivity of SMA was diminished in the areas of ISS. These results suggested that ISS does not have the characteristics of nerves, muscle or blood vessels, but have the same character as the lympho-endothelium.

Correlation of ISS and clinicopathological variables. Each clinicopathological variable was compared based on ISS by the univariate analysis (Table I). A significant correlation was observed between ISS and tumor size $(\mathrm{p}=0.001)$, lymph node status $(\mathrm{p}<0.001)$, negative estrogen receptor $(\mathrm{ER})$ expression $(\mathrm{p}=0.050)$, positive VEGF-C expression $(\mathrm{p}<0.001)$ and disease recurrence $(\mathrm{p}<0.001)$. However, based on the multivariate logistic regression analysis, only lymph node status $(\mathrm{p}=0.024$, $\mathrm{OR}=2.841)$ and disease recurrence $(\mathrm{p}=0.008, \mathrm{OR}=3.831)$ remained independent factors correlating with ISS. These 


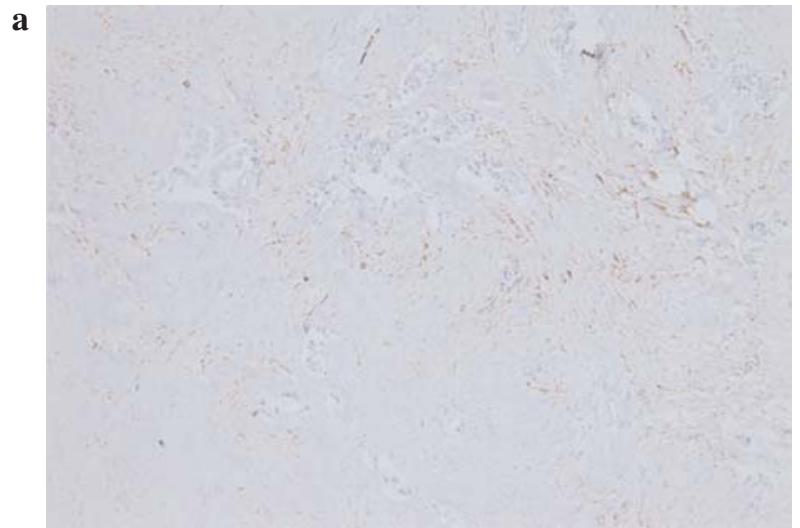

c
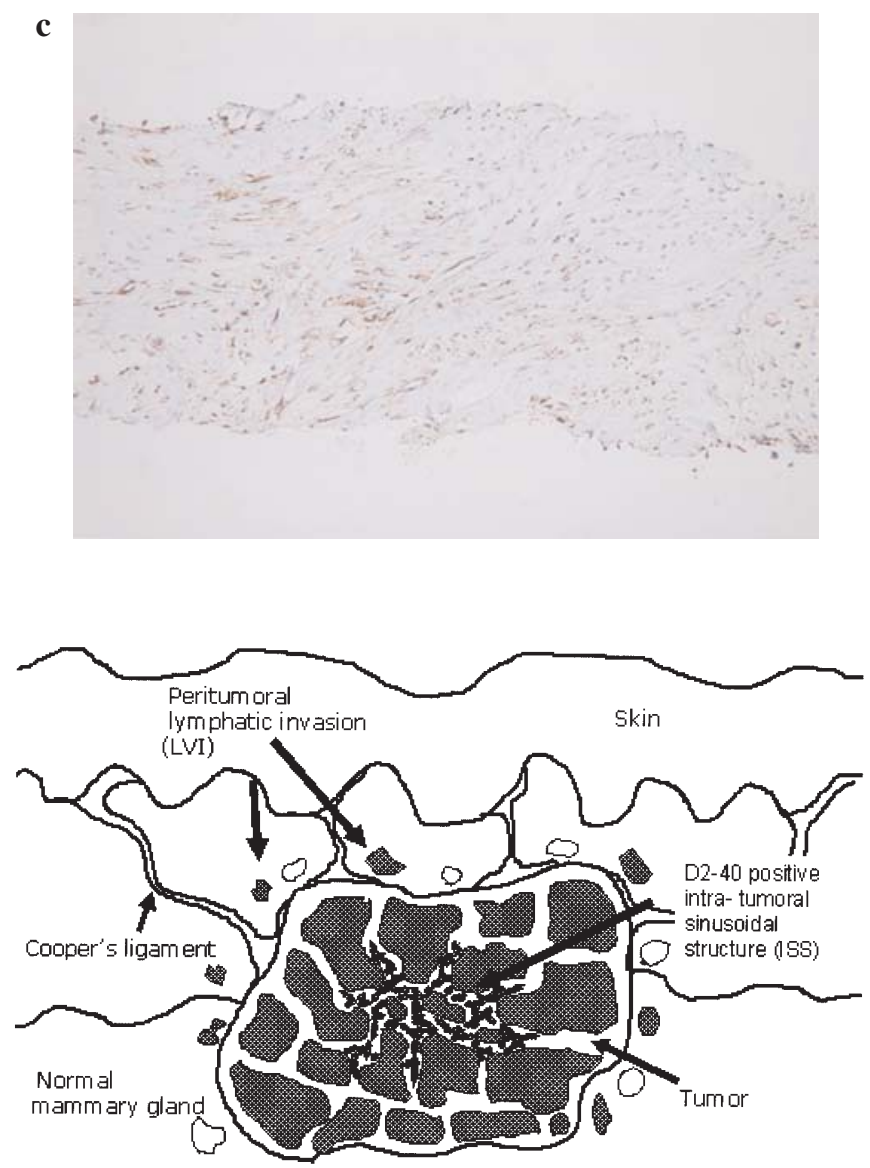

Figure 2. Schematic figure of ISS. D2-LVI was observed mainly in the peritumoral area, whereas ISS was observed mainly in the central tumor area.

correlations were also demonstrated when cases with T1 tumor were selected and analyzed. In the univariate analysis, significant correlations were observed between ISS and lymph node status $(\mathrm{p}<0.001)$, or disease recurrence $(\mathrm{p}=0.001)$ in 45 cases, with a tumor of $<2 \mathrm{~cm}$ in diameter (Table II).

To determine the possible involvement of ISS in disease progression through lymphatic systems, we analyzed factors affecting lymph node status or disease recurrence by multivariate analysis. ISS ( $\mathrm{p}=0.001, \mathrm{OR}=4.150$ ) was the strongest factor affecting LN metastases, followed by VEGF-C $(\mathrm{p}=0.024, \mathrm{OR}=2.786)$, and the correlation was stronger than that with LVI (Table III). A significant correlation was

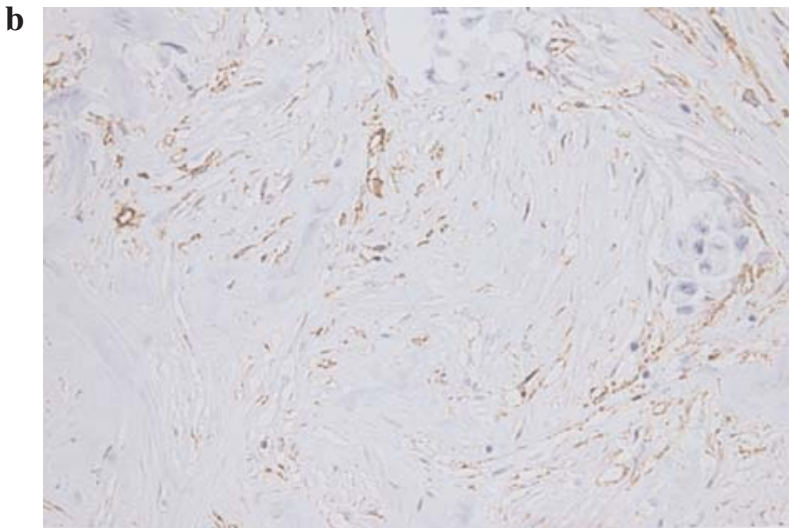

Figure 1. An example of ISS which shows border indistinctness in stroma between tumor lobulations accompanied with fibrous bands at low magnification (a). At high magnification, this expression consists of small, deformed, various-sized sinusoids, lined with D2-40 positive endothelia (b). ISS was observed pre-operatively by CNB specimen (c).

Table II. Correlation between ISS and lymph node status/ disease recurrence in cases with a tumor diameter of $<2 \mathrm{~cm}$.

\begin{tabular}{lrrr}
\hline & \multicolumn{2}{c}{ ISS } & \\
\cline { 2 - 3 } & - & + & p-value \\
\hline Lymph node status & & & \\
- & 28 & 2 & \\
+ & 5 & 10 & $<0.001$ \\
Disease recurrence & & & \\
No & 32 & 6 & \\
Yes & 1 & 6 & 0.001 \\
\hline
\end{tabular}

displayed between disease recurrence and D2-LVI $(\mathrm{p}<0.001$, $\mathrm{OR}=10.317)$, lymph node status $(\mathrm{p}<0.001, \mathrm{OR}=7.092)$, tumor size $(p=0.001, O R=8.758)$ and ISS $(p=0.003, O R=5.102)$. Furthermore, a significant difference in disease-free survival was found between patients with and without ISS $(\mathrm{p}<0.0001$, log-rank test) (Fig. 4a). A significant difference in overall survival was also found between patients with and without ISS (Fig. 4b).

Evaluations of ISS by biopsy specimens. In an analysis of 45 CNB specimens and postoperative specimens, significant correlations were observed between the evaluation of ISS using CNB specimens and that using postoperative specimens $(\mathrm{p}=0.002)$ (Table IV). Moreover, there was a significant 
a

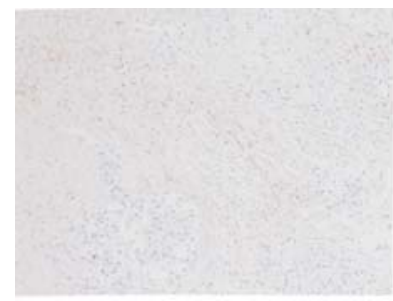

D2 -40

c

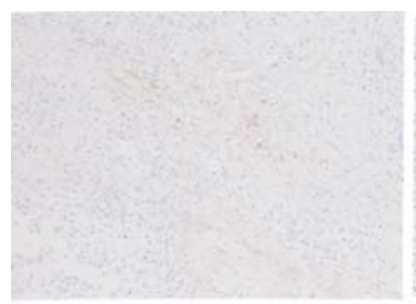

D2 -40

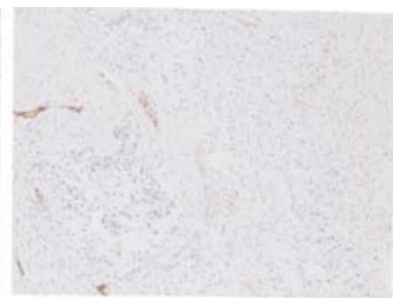

CD34

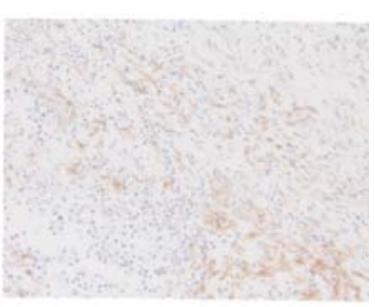

SMA b
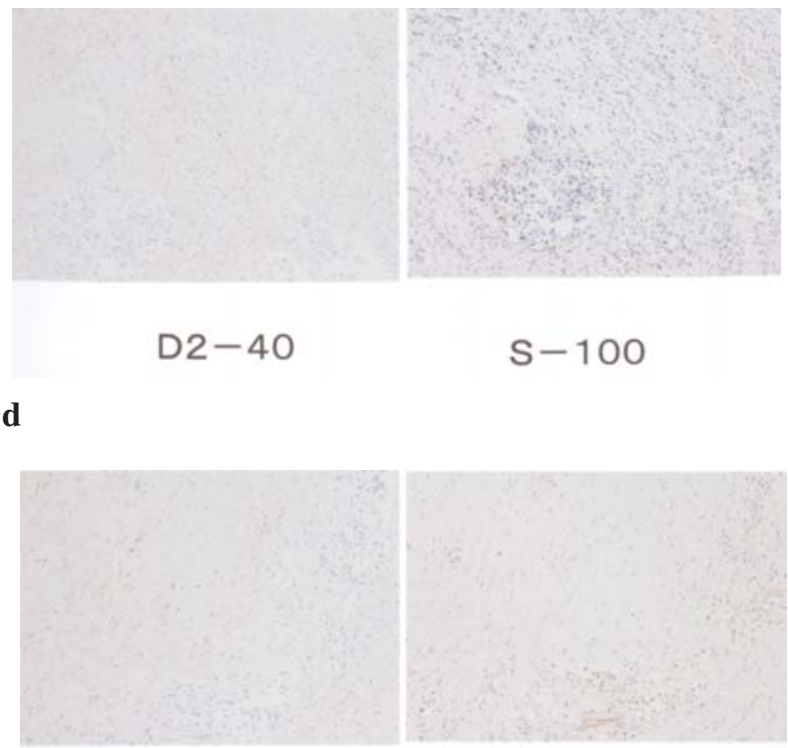

$\mathrm{D} 2-40$

Figure 3. Symmetric 'mirror image' specimens were made from the same surface and stained by CD34 (a), S-100 (b), SMA (c), and Flt-4 (d). The ISS area did not react with CD34, S100 or Flt-4. Although the tumor stroma was diffusely stained with SMA, its immunoreactivity was diminished in the areas of ISS.
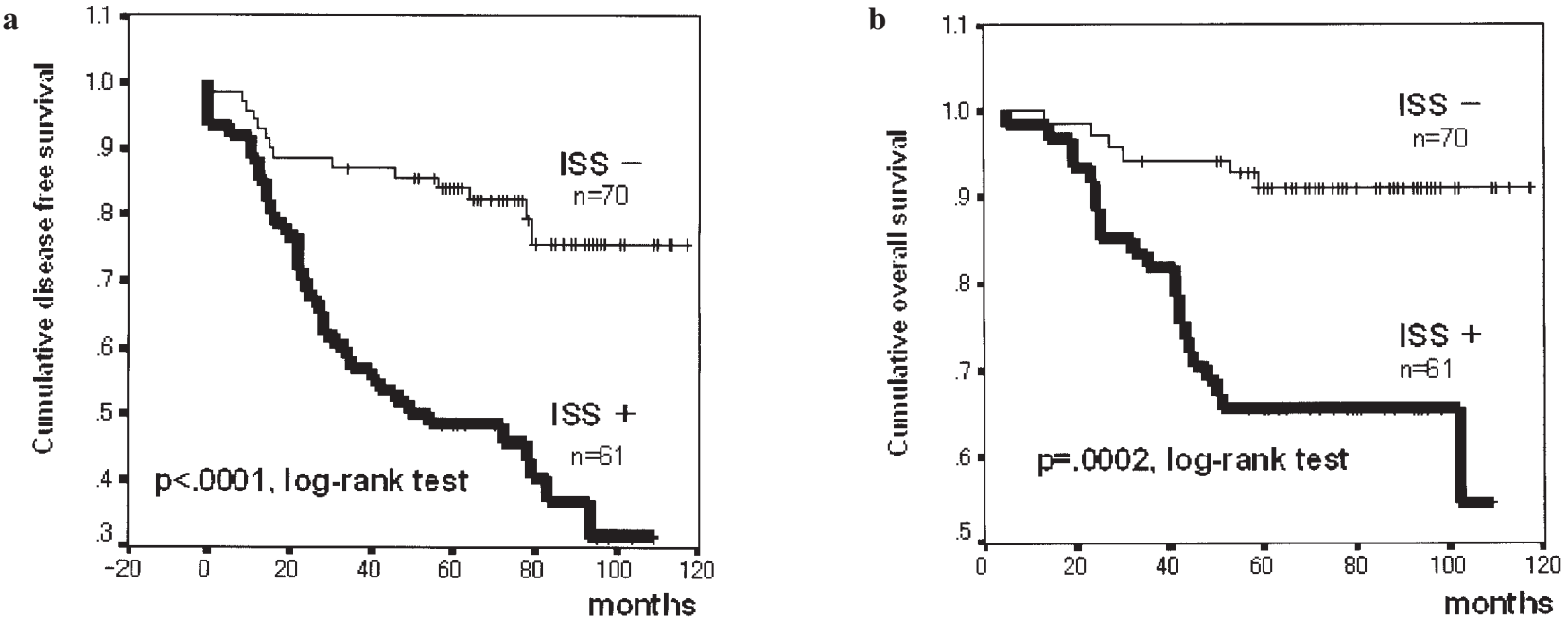

Figure 4. Kaplan-Meier survival analysis of DFS (a) and OS (b) depending on the ISS status. The ISS status exhibited a significantly worse DFS and OS compared with D2-TLS (-) (DFS, $\mathrm{p}<0.0001$; OS, $\mathrm{p}=0.0002$ and log-rank test, respectively).

correlation between positive ISS evaluated by CNB and positive lymph node status $(\mathrm{p}=0.020)$.

\section{Discussion}

Previous studies have shown several molecules critical for lymphatic vessel development in humans (22). Techniques to specifically detect the lymphatic endothelium using these markers have been developed. D2-40 is one of these markers, and detects an epitope on an O-linked sialoglycoprotein specifically expressed in the lymphatic endothelium (20). Several studies have investigated the involvement of lymphatic systems in breast cancer with these specific markers and found significant clinical importance of immunohistochemical detection of LVI in this disease. According to previous reports, the determination of LVI by immunohistochemical detection demonstrated the importance of LVI as an independent prognostic factor correlating with lymphatic progression and survival, compared to that by conventional H\&E-based detection $(16,17,23)$. As these detailed examinations have 
Table III. Correlation between disease recurrence/lymph node status and clinicopathological variables in multivariate analysis.

\begin{tabular}{|c|c|c|c|c|c|c|}
\hline \multirow[b]{2}{*}{ Variable } & \multicolumn{3}{|c|}{ Recurrence } & \multicolumn{3}{|c|}{ Lymph node status } \\
\hline & Odds ratio & $95 \% \mathrm{CI}$ & $\mathrm{p}$-value & Odds ratio & $95 \% \mathrm{CI}$ & p-value \\
\hline $\begin{array}{l}\text { Age } \\
\leq 50 \\
>50\end{array}$ & 0.668 & $0.248-9.029$ & NS & 0.971 & $0.195-4.821$ & NS \\
\hline $\begin{array}{c}\text { Tumor size } \\
\leq 20 \mathrm{~mm} \\
>20 \mathrm{~mm}\end{array}$ & 8.758 & $2.392-32.063$ & 0.001 & 1.922 & $0.776-4.762$ & NS \\
\hline $\begin{array}{l}\text { Menopause } \\
\text { No } \\
\text { Yes }\end{array}$ & 0.204 & $0.029-1.432$ & NS & 0.887 & $0.211-6.010$ & NS \\
\hline $\begin{array}{l}\text { Lymph nod } \\
\text { Negative } \\
\text { Positive }\end{array}$ & 7.092 & $0.047-0.418$ & $<.001$ & & & \\
\hline $\begin{array}{l}\text { ER } \\
\text { Negative } \\
\text { Positive }\end{array}$ & 0.999 & $0.375-2.661$ & NS & 1.580 & $0.270-1.480$ & NS \\
\hline $\begin{array}{l}\text { Nuclear gra } \\
1 \\
2 \text { or } 3\end{array}$ & 0.494 & $0.542-7.578$ & NS & 0.726 & $0.232-2.269$ & NS \\
\hline HE-LVI & 2.801 & $0.114-1.111$ & NS & 1.823 & $0.758-4.382$ & NS \\
\hline D2-LVI & 10.317 & 2.831-37.596 & $<.001$ & 1.622 & $0.650-4.049$ & NS \\
\hline VEGF-C & 0.956 & $0.302-3.622$ & NS & 2.786 & $0.148-0.375$ & 0.024 \\
\hline ISS & 5.102 & $0.066-0.580$ & 0.003 & 4.150 & $0.103-0.564$ & 0.001 \\
\hline
\end{tabular}

Table IV. Correlation between ISS in CNB specimens and lymph node status/ISS in postoperative specimens.

\begin{tabular}{lrrr}
\hline & \multicolumn{2}{c}{ ISS in CNB } & \\
\cline { 2 - 3 } & - & + & p-value \\
\hline Postoperative specimen & & & \\
$\quad$ ISS- & 17 & 5 & \\
ISS+ & 7 & 16 & 0.002 \\
Lymph node status & & & \\
- & 14 & 5 & \\
+ & 10 & 16 & 0.02 \\
\hline
\end{tabular}

indicated, the presence of LVI was newly added as one of the major factors for determining treatment plans in the consensus meeting of St. Gallen in 2005 (7).

According to these reports, including our previous study, lymphatic vessels were mainly observed in the peritumoral area. At the same time, some reports mentioned immunoreactive lymphatic endothelial structures intra-tumorally $(11,23)$ or in the tumor stroma $(14,18)$. As shown in the present study, these structures were not detected by H\&E staining and differed morphologically from the normally developed lymphatic vessels. However, ISS specifically reacted with D2-40, but not CD34, S-100, Flt-4 and SMA. Therefore, in the present study we attempted to independently evaluate these intra-tumoral sinusoidal structures showing lympho-endothelial immunoreactivity.

We observed certain characteristics of ISS that are different from those of normal lymphatic systems. First, morphologically ISS could not be distinguished from the surrounding tumor stroma by H\&E staining. It did not react with CD34, S-100, Flt-4 or SMA. This D2-40-specific immunoreactive structure showed a similar staining pattern to the lymphatic vessels that were elongated and flat endothelial fragments with a completed vessel was scarcely observed. Moreover, it was difficult to show any connection from ISS to the lymphatic vessels outside of the tumor. Second, cancerous infiltration was commonly observed in ISS. ISS was commonly observed between the lobulations of cancer nests and were accompanied by thick fibrous tissues. In other words, intimate contact was displayed between ISS and cancer cells. Finally, although ISS correlated with tumor size and nodal status, no correlation was demonstrated between the existence of ISS and LVI. Thus, ISS seemed different from normally developed lymphatic vessels. However, at the same time, several observations indicated that ISS may have lymph vessel-like characteristics 
such as ISS being stained selectively by D2-40, having a significant correlation with VEGF-C expression of the cancer cells and being present in combination with tumor stroma. Furthermore, ISS was observed in cases of a small tumor, suggesting that ISS was not a by-product secondary to the tumor progression, but was already formed in early-stage tumors. From these observations, therefore, we assumed that ISS was a unique sinusoidal structure that differs from lymphatic systems, or can coexist with tumor stroma and feeble or destroyed lymphatic vessels. However, it was difficult to determine the pathogenesis or the function of ISS from these analyses. Therefore, we tried to investigate the clinical significance of ISS by comparing it with clinicopathological factors.

In the present study, we found that the existence of ISS was significantly correlated with tumor size and lymph node status, two major factors that determine disease prognosis. Furthermore, the multivariate analysis demonstrated that ISS independently correlated with lymph node status and disease recurrence. Most importantly, ISS had the strongest correlation with nodal status compared to other factors, preceding $\mathrm{T}$ or LVI. In terms of the prognosis of the disease, ISS was listed as one of the most powerful independent prognostic factors in line with other well-known conventional prognostic factors, such as tumor size, nodal status and LVI. Those results strongly suggested that ISS was deeply involved in the lymphatic spread and played an important role in the disease progression of breast cancer.

Williams et al (13) reported that naturally occurring breast carcinomas invade and destroy lymph vessels rather than promote their proliferation. Agarwal et al (18) reported that lymphatic vessels were seen in the intra-tumoral area adjacent to pre-existing normal lobules and ducts, which were interpreted as pre-existing vessels 'entrapped' within the tumor. However, from our observations in the present study, the clinical significance of these ISS was much more important than the simple 'entrapped' ducts of Agarwal's hypothesis. Although the existence of ISS correlated well with tumor size, significant correlations were also observed between ISS and lymph node status or disease recurrence, even in cases of a small tumor diameter of $<2 \mathrm{~cm}$. This suggests their important role in determining disease progression and prognosis from the early stages of breast cancer. We assume that part of the lymphatic systems is not only 'entrapped', but also that novel lymphatic systems are generated as ISS in the tumor, because there was a significant correlation between ISS and VEGF-C expression.

The most important point in our present study is the fact that ISS could be judged by core-needle biopsy specimens. The conventional method of determining LVI by H\&E staining requires the whole tumor including normal breast tissues outside the tumor, and a well-trained pathologist. As shown in the results, the ISS of the tumor was accurately determined by only a piece of a biopsy specimen. Moreover, the existence of ISS in the biopsy sample may precisely predict lymph node metastasis or disease recurrence preoperatively. In addition, it may offer useful information in deciding between the application of sentinel lymph node biopsy or systemic therapy before operation, because of the intimate correlation between ISS and the lymphatic spread of the disease.
In conclusion, ISS plays a crucial role in disease progression through lymphatic systems in breast cancer. Our data showed that ISS in tumors were determined by a piece of a biopsy specimen and could predict the prognosis as accurately as a whole tumor examination. Evaluation of ISS involves only a simple, standardized immunohistochemical technique that could easily be added as routine work. Therefore, it may be a useful evaluation method to apply in clinical settings to determine the treatment plan before operation or without operation.

\section{Acknowledgements}

We thank Mr. Kazuto Miyazaki for his expert technical assistance.

\section{References}

1. Khonji N, Clarke D and Mansel RE: Breast Cancer Metastasis. In: Cancer Metastasis, Molecular and Cellular Mechanisms and Clinical Intervention. Jiang WG and Mansel RE (eds). Kluwer Academic Publishers, Norwell, MA, Chapter 13, 2000.

2. Carter CL, Allen C and Henson DE: Relation of tumor size, lymph node status and survival in 24740 breast cancer cases. Cancer 63: 181-187, 1989.

3. Fisher B, Bauer M, Wickerham DL, Redmond CK and Fisher ER: Relation of number of positive axillary nodes to the prognosis of patients with primary breast cancer. An NSABP update. Cancer 52: 1551-1557, 1983.

4. Takashima T, Onoda N, Ishikawa T, et al: Proliferating cell nuclear antigen labeling index and p53 expression predict outcome for breast cancer patients with four or more lymph node metastasis. Int J Mol Med 8: 159-163, 2001.

5. Takashima T, Onoda N, Ishikawa T, et al: Prognostic value of combined analysis of estrogen receptor status and cellular proliferative activity in breast cancer patients with extensive lymph node metastasis. Oncol Rep 9: 589-594, 2002.

6. Early Breast Cancer Trialist's Collaborative Group. Polychemotherapy for early breast cancer: An overview of the randomized trials. Early Breast Cancer Trialist's Collaborative Group. Lancet 352: 930-942, 1998

7. Goldhirsch A, Glick JH, Gelber RD, et al: Meeting highlights: International expert consensus on the primary therapy of early breast cancer. Ann Oncol 16: 1569-1583, 2005.

8. Skobe M, Hawighorst T, Jackson DG, et al: Induction of tumor lymphangiogenesis by VEGF-C promotes breast cancer metastasis. Nature Med 7: 192-198, 2001.

9. Choi WW, Lewis MM, Lawson D, et al: Angiogenic and lymphangiogenic microvessel density in breast carcinoma: correlation with clinicopathologic parameters and VEGF-family gene expression. Mod Pathol 18: 143-152, 2005.

10. Nakamura Y, Yasuoka H, Tsujimoto M, et al: Clinicopathological significance of vascular endothelial growth factor-C in breast carcinoma with long-term follow-up. Mod Pathol 16: 309-314, 2003.

11. Nakamura Y, Yasuoka H, Tsujimoto $\mathrm{M}$, et al: Lymph vessel density correlates with nodal status, VEGF-C expression and prognosis in breast cancer. Breast Cancer Res Treat 91: 125-132, 2005.

12. Vleugel MM, Bos R, Van der Groep P, et al: Lack of lymphangiogenesis during breast carcinogenesis. J Clin Pathol 57: 746-751, 2004.

13. Williams CS, Leek RD, Robson AM, et al: Absence of lymphangiogenesis and intratumoral lymph vessels in human metastatic breast cancer. J Pathol 200: 195-206, 2003.

14. Schoppmann SF, Bayer G, Aumayr K, et al: Prognostic value of lymphangiogenesis and lymphovascular invasion in invasive breast cancer. Ann Surg 240: 306-312, 2004.

15. Kato T, Prevo R, Steers G, et al: A quantitative analysis of lymphatic vessels in human breast cancer, based on LYVE-1 immunoreactivity. Br J Cancer 93: 1168-1174, 2005.

16. Tezuka K, Onoda N, Takashima T, et al: Lymphatic vessel infiltration in combination with micro vessel density predicts breast cancer patients - Preliminary study with immunohistochemistry. J Clin Exp Med 217: 1139-1140, 2006 (In Japanese). 
17. Tezuka K, Onoda N, Takashima T, et al: Prognostic significance of lymphovascular invasion diagnosed by lymphatic endothelium immunostaining in breast cancer patients. Oncol Rep 17: 997-1003, 2007.

18. Agarwal B, Saxena R, Morimiya A, Mehrotra S and Badve S: Lymphangiogenesis does not occur in breast cancer. Am J Surg Pathol 29: 1449-1455, 2005.

19. Kahn HJ and Marks A: A new monoclonal antibody, D2-40, for detection of lymphatic invasion in primary tumors. Lab Invest 82: 1255-1257, 2002.

20. Kahn HJ, Bailey P and Marks A: Monoclonal antibody D2-40, a new marker of lymphatic endothelium, reacts with Kaposi's sarcoma and a subset of angiosarcomas. Mod Pathol 15: 434-400, 2002.
21. Evangelou E, Kyzas PA and Trikalinos T: Comparison of the diagnostic accuracy of lymphatic endothelium markers: Bayesian approach. Mod Pathol 18: 1490-1497, 2005.

22. Forkman J and Kaipainen A: Genes tell lymphatics to sprout or not. Nature Immunol 5: 11-12, 2004.

23. Van den Eynden GG, Van der Auwera I, Van Laere SJ, et al: Distinguishing blood and lymph vessel invasion in breast cancer: a prospective immunohistochemical study. Br J Cancer 94: 1643-1649, 2006. 